УДК 622.281 .74

\title{
АНАЛИЗ ТИПОВЫХ ФОРМ ДЕФОРМАЦИЙ ПРИКОНТУРНОГО УГЛЕПОРОДНОГО МАССИВА И ОСОБЕННОСТИ ВОССТАНОВЛЕНИЯ КРЕПЛЕНИЯ ГОРНЫХ ВЫРАБОТОК
}

\author{
Цибаев Сергей Сергеевич 1 , \\ cibaevss@kuzstu.ru
}

\section{Ренев Алексей Агафангелович1, raa@kuzstu.ru}

\section{Ванг Цин²,}

qwang@sdust.edu.cn

\author{
Юй Фэн-Хай², \\ yufenghai2006@163.com
}

\author{
1 Кузбасский государственный технический университет имени Т.Ф. Горбачева, \\ Россия, 650000, г. Кемерово, ул. Весенняя, 28. \\ 2 Шаньдунский научно-технический университет, \\ КНР, 266590, г. Циндао, Шоссе Циеньваньган, 579.
}

\begin{abstract}
Актуальность. В настоящее время более 75 \% проводимых подземных горных выработок поддерживаются при помощи анкерного крепления. В процессе длительной эксплуатации выработок возникают деформации приконтурного массива пород и элементов крепи. Актуальной научно-практической задачей представляется выявление типовых форм деформаций пород и элементов крепи с целью разработки проектных решений и обоснования параметров крепи при производстве текущего ремонта или восстановления подземных выработок.

Цель: разработка проектных решений и обоснование параметров анкерного крепления при ремонте и восстановлении подземных горных выработок.

Методы: натурные исследования, статистическая обработка результатов экспериментальных исследований.

Результаты. Приведено описание, причины возникновения и формы проявления геомеханических процессов в приконтурном уәлепородном массиве горных выработок, закрепленных анкерной крепью. На основе длительных натурных наблюдений установлено шесть форм типовых деформаций приконтурного массива. Процесс сводообразования в кровле выработок начинается с разуплотнения приконутрного слоя, которое служит причиной расслоения пород под действием гравитационных сил, а также образования полостей. Отжим угля из боков выработок и вывалы угля происходят в связи с образованием вокруг выработки зон неупругих деформаций, где уголь и породы находятся в несвязанном состоянии и удерживаются в равновесии за счёт сил трения и отпора крепи. Ширина зоны неупругих деформаций зависит от многих факторов. Существенное влияние оказывают ширина выработки и угол внутреннего трения. При увеличении ширины выработки увеличивается коэфффициент концентрации напряжений в боках. Разработаны проектные решения по усилению крепления или полной перекрепке поврежденных участков горных выработок при геомеханических процессах локального вывалообразования. Они включают в себя: схему установки, обоснование параметров и номенклатуру применяемых элементов крепи-усиления, технологию работ, список применяемого оборудования.
\end{abstract}

Ключевые слова:

Устойчивость горных выработок, ремонт горных выработок, анкерное крепление, деформации, смещения, конвергенция.

\section{Введение}

Важнейшей проблемой безопасного длительного поддержания подземных горных выработок является оценка влияния техногенного воздействия на углепородный массив и элементы крепления (затопление горных выработок, воздействие сейсмических и ударных воздушных волн от массовых промышленных и неконтролируемых взрывов). За последние 20 лет на шахтах Кузбасса произошло 13 аварий с групповыми несчастными случаями со смертельным исходом. Большинство эпизодов связаны с взрывами газовоздушной смеси и угольной пыли, сопровождающимися распространением сейсмических и ударных волн, а также резким кратковременным повышением температуры в зоне взрыва. При ликвидации последствий техногенных аварий, вызывающих образование подземных пожаров, а также при консервации или ликвидации угольных шахт «мокрым» способом вода заполняет сеть горных выработок на полное сечение. Совместная разработка угольных пластов открытым и подземным способами в долгосрочной перспективе приводит к деформациям углепородного массива и нарушению работоспособности анкерной крепи [1-4]. При этом в действующих нормативных и литературных источниках отсутствует дифференцированный подход к определению характерных деформаций приконтурного улепородного массива.

Объектом исследования выступила область углепородного массива вокруг горных выработок в условиях предприятий ПАО «Распадская» (ш. «Распадская»), ОАО УК «Южный Кузбасс» (ш. «им. В.И. Ленина), АО «СУЭК-Кузбасс» (ш. «им. В.Д. Ялевского», ШУ «Талдинское-Западное»), АО ХК «СДС-Уголь» (ш. «Южная»). Исследования проводятся в рамках выполнения государственного задания № 075-03-2021-138/3. 


\section{Результаты натурных исследований}

На основании результатов продолжительных наблюдений за состоянием приконтурного массива пород и элементов анкерного крепления [5-9] было установлено, что геомеханические процессы в приконтурных слоях выработок происходят в следующей последовательности. Процесс сводообразования вызыван в первую очередь разуплотнением пород. Разуплотнение состоит в образовании по контуру выработки зоны повышенной трещиноватости в результате раскрытия естественных трещин и создания новых трещин. Трещины разуплотнения развиваются параллельно обнаженной поверхности пород, образуя зоны ослабления. В результате разуплотнения происходит расслоение пород. Процесс расслоения определяется двумя факторами - трещиноватостью и слоистостью [10-12]. Происходит разделение пород на отдельности, которые под действием гравитационных сил смещаются в сторону выработки. При расслоении пород отдельные слои, в основном нижележащие, отслаиваются от верхних слоев, то есть происходит процесс отслоения отдельностей от массива и их смещение к контуру выработки. Отслоившиеся породы скапливаются на решетчатой затяжке, нагружая решетчатую затяжку и анкеры. В результате деформаций решетчатой затяжки и подхватов происходит обрушение отслоившихся пород в выработку. Расслоение пород в кровле выработок распространяется за пределы границ выработок по ширине. В результате возрастает напряжение в боках выработок и деформация угля. Происходят отжимы, вывалы угля из боков.

По результатам натурных наблюдений было выявлено шесть основных типовых форм деформирования приконтурного углепородного массива.

При скоплении отслоившихся пород кровли на решетчатой затяжке без ее деформации (рис. 1) приконтурные слои кровли деформируются постепенно, процесс развивается снизу вверх, вглубь пород кровли.

При расслоении пород нижние слои, теряя связь с вышележащими, смещаются и ложатся на решетчатую затяжку. Происходит нагружение решетчатой затяжки, подхватов и анкеров. Высота обрушенных пород в основном изменяется от 0,1 до 0,5 м. Состояние решетчатой затяжки и подхватов зависит от фактической толщины слоя отслоившихся пород. При провисаниях несущая способность решетчатой затяжки снижается. Таким образом, фактическая несущая способность решетчатой затяжки определяется диаметром прутка, размерами ячейки и стрелой провисания. При провисании решетчатой затяжки CC-4, CC-5, CC6 на величину 0,4 м целесообразно производить ремонт на таких участках. Ремонт заключается в выпуске разрушенных пород, закреплении кровли и заполнении пустоты после выпуска пород.

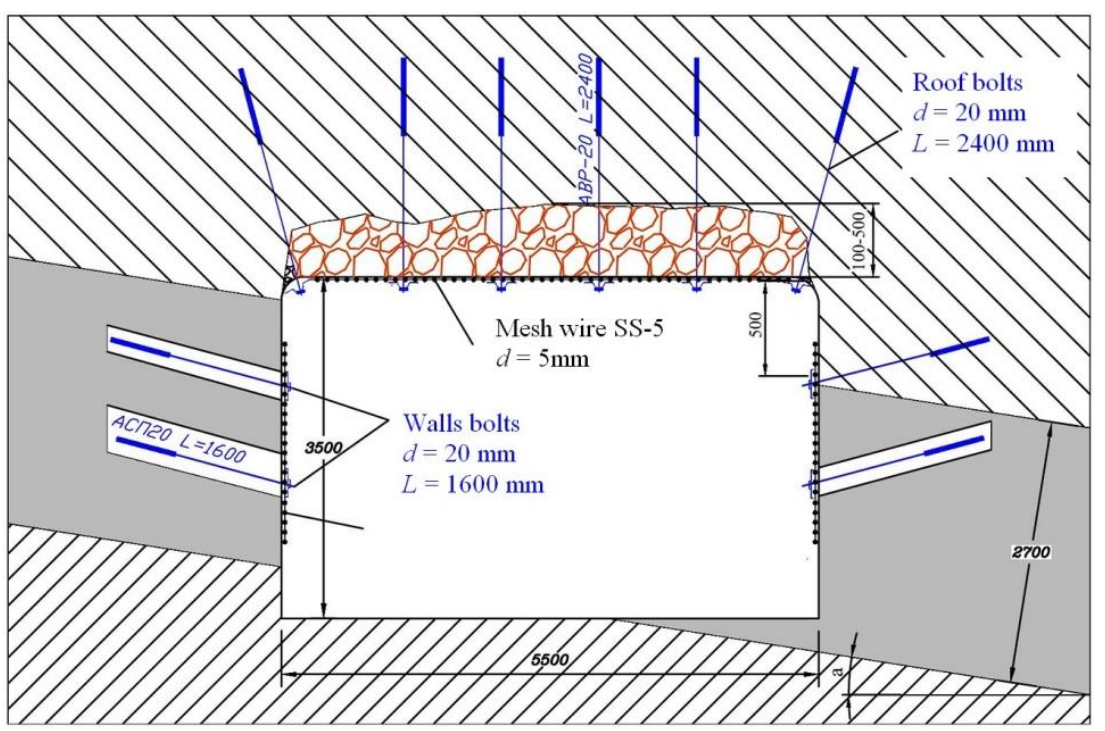

Pис. 1. Скопление отслоившихся пород кровли на решетчатой затяжке без ее деформации

Fig. 1. Accumulation of exfoliated roof rocks on mesh wire without its deformation

При скоплении отслочвшихся пород кровли с деформацией решетчатой затяжки и образованием вывалов и куполов (рис. 2) на участках с разорванной решеткой происходят прорывы пород в выработку. Образовавшиеся вывалы имеют разную форму и параметры.

Разрывы решетчатой затяжки с вывалами пород чаще всего наблюдаются на локальных участках, где породы ослаблены, имеется повышенная трещиноватость, повышенная влажность пород, вблизи зон с дизъюнктивной нарушенностью [13]. Установлено, что разрывы решетчатой затяжки происходят при скоплении отслоившихся пород высотой 0,5 м и более. Участки с разорванной решетчатой затяжкой и наличием отслоившихся пород на прилегающих к вывалу смежных участках подлежат срочному ремонту. Ремонт осуществляется путем усиления крепления смежных участков поддерживающей крепью, выпуска пород вблизи с вывалом, закрепления вывала анкерной крепью и заполнения образовавшейся пустоты. 
Заполнение может производиться лесом, вспенивающимися смолами, пенобетоном с последующим возведением решетчатой затяжки. При разрывах решет- чатой затяжки на участках, где происходят процессы сплошного сводообразования, вывалы в дальнейшем развиваются в длинные и высокие купола.

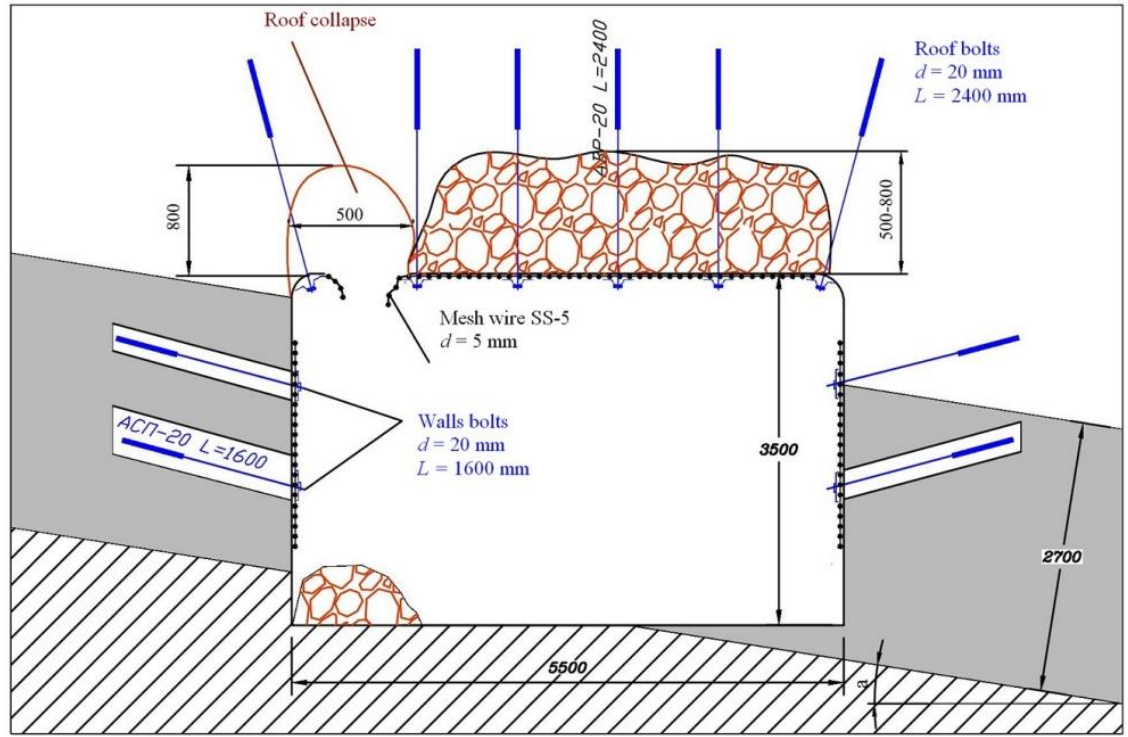

Рис. 2. Скопление отслоившихся пород кровли с деформацией решетчатой затяжки и образованием вывалов и куполов

Fig. 2. Accumulation of exfoliated roof rocks with mesh wire deformation and formation of collapses and domes

При скоплении обрушенных пород с деформащчией решетчатой затяжки и образованием купола по всей ширине выработки (рис. 3) высота куполов, как пра- вило, составляет до 2,5-3,0 м, ширина равна ширине выработки, а протяженность может достигать 10 м.

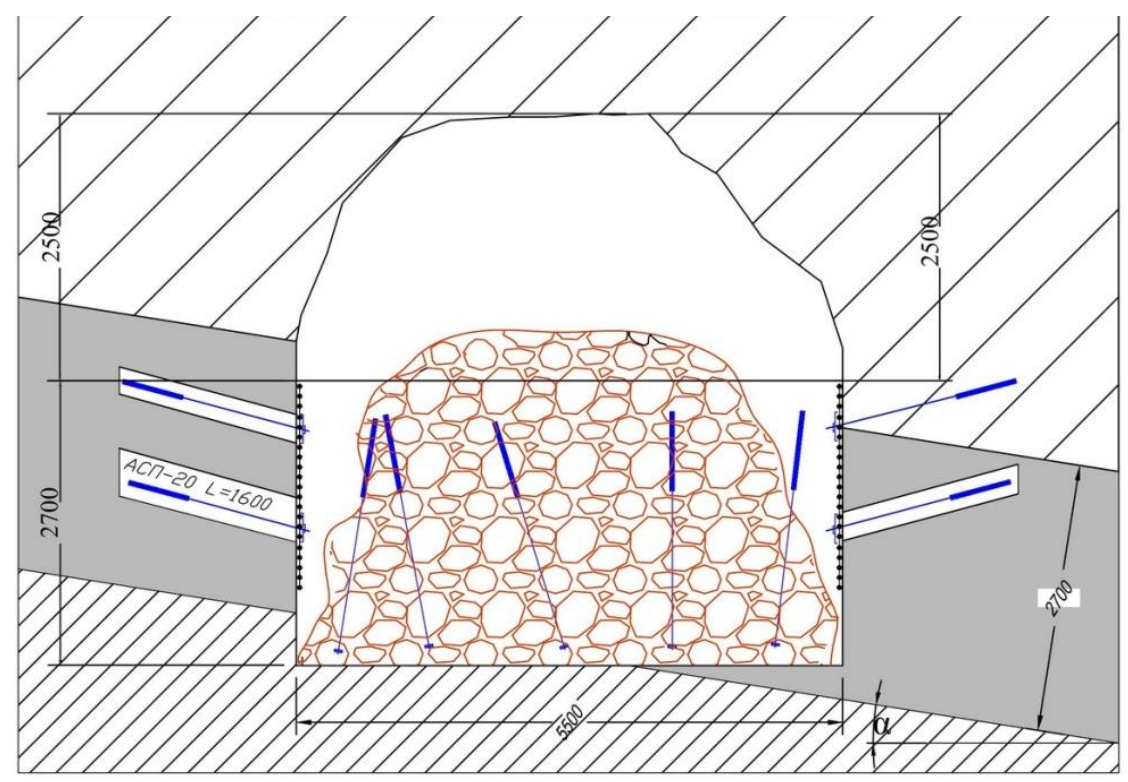

Рис. 3. Скопление обрушенных пород с деформацией решетчатой затяжки и образованием купола по всей ширине штрека

Fig. 3. Accumulation of collapsed rocks with mesh wire deformation and dome formation along the entire width of the roadway

При данном виде деформаций массива на аварийных участках производится полная перекрепка выработки. Работы по перекрепке выполняются со специального полка. Обрушившиеся породы выпускаются из под решетки в выработку, убираются деформированные части решетчатой затяжки, за- тем производится оборка боков и кровли. По сечению выработки устанавливается либо новая анкерная крепь, либо металлическая рамная крепь. Пустоты в боках и кровле заполняются либо выкладкой деревянной костровой крепи, либо пенобетоном (текфом). 
При отжиме пород в боках выработок в форме призмы сползания (рис. 4) углы призмы сползания $\theta$ изменяются в широких пределах - от $50^{\circ}$ до $65^{\circ}$, а ширина призмы сползания $C$ - от 1,0 до 1,8 м. Отжим в виде призм сползания вызывает значительные обнажения пород кровли и их вывалы в выработку. Ширина выработок вследствие этого на контакте с породами резко увеличивается. При отсутствии вывалов породы из кровли на участках выработок с отжимом производится усиление крепи исходя из ширины призмы сползания. Первоначально подводится решетчатая затяжка, затем устанавливается усиливающая анкерная крепь.

Отжим угля и пород в боках выработок в виде вызвалов (рис. 5) наблюдается на участках, где кровля труднообрушаемая.

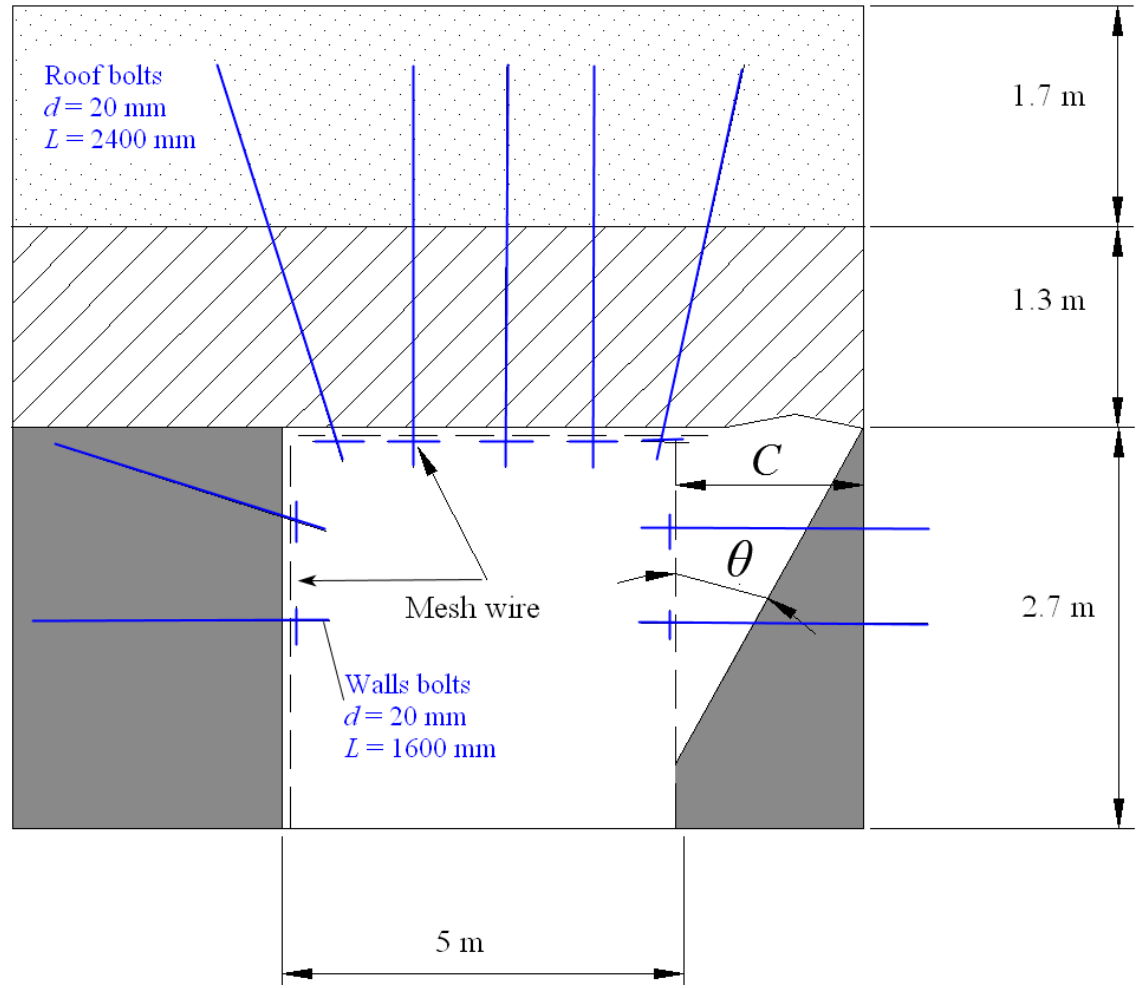

Pис. 4. Отжим угля из бока вентиляиионного штрека 4-9-25 в виде призмы сползания в условиях ш. «Распадская» Fig. 4. Squeezing of rocks in the walls of air roadway 4-9-25 of «Raspadskaya» mine in the form of a sliding prism

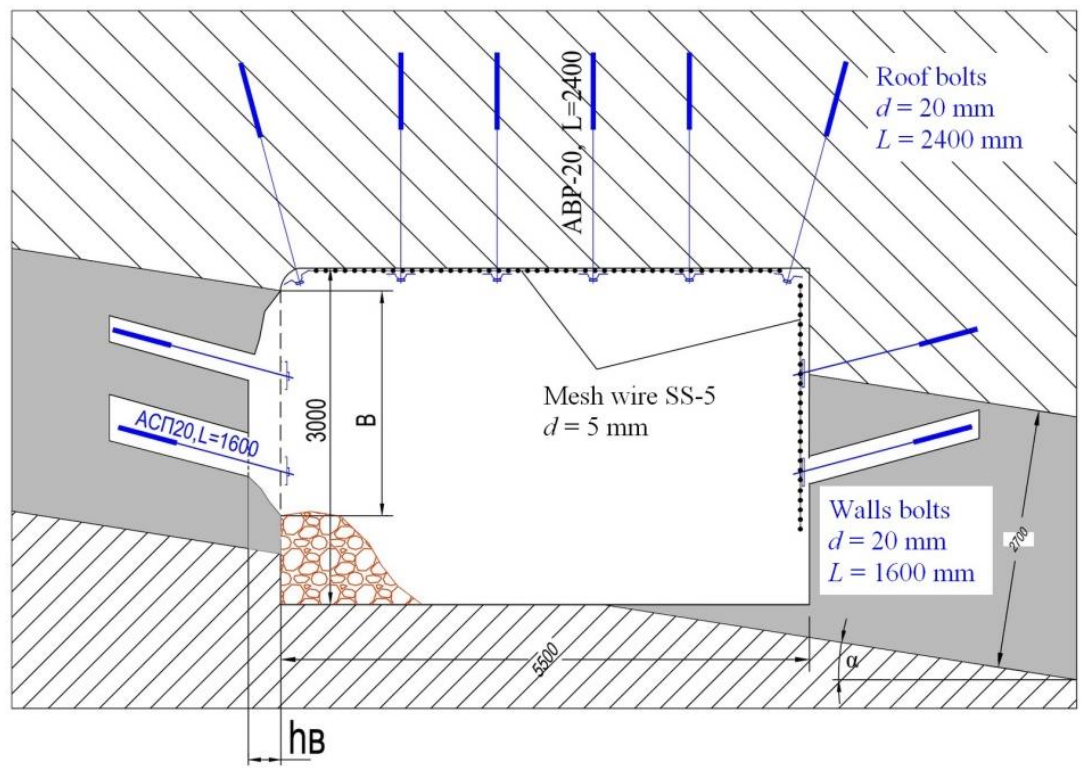

Pис. 5. Отжим угля и пород в боках выработок в виде вывалов

Fig. 5. Squeezing of coal and rocks in the walls of workings in the form of dumps 


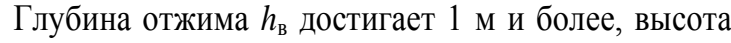
$B$ достигает высоты выработки. Отжимы, как правило, локализуются в подготовительных выработках со стороны висячего бока. При незначительных вывалах в боках ремонтные работы производятся установкой и закреплением решетчатой затяжки или полимерной сетки с помощью одного-двух сталеполимерных анкеров. При глубоких и высоких вывалах производится перекрепка участка. Убирается отслоившийся уголь и порода, выравнивается поверхность вывала, возводится решетчатая затяжка и анкеры. В отдельных случаях вывалы заполняются скрепляющими составами типа Карбофил, Геофом, Текфом.

Эпизоды пластического выдавливания угля и пород из боков выработок (рис. 6) происходят в выработках с труднообрушаемой кровлей на участках с ослабленными прочностными свойствами угля. Ослабленные слои угля при высоких горизонтальных напряжениях выдавливаются в сторону выработок. Выдавленная часть угля в выработку разрушается, нарушается крепление боков. Чаще выдавливание угля наблюдается в выработках со стороны висячего бока. Величина выдавливания угля $d$ в выработку составляет 0,5-0,6 м, иногда больше. При выдавливании угля в выработках образуются нависающие карнизы. При высоких выработках они представляют опасность. Поэтому на таких участках производится перекрепка боков. Разрушенный уголь обрушается, бок выравнивается, перекрывается решетчатой затяжкой или полимерной сеткой, возводятся анкеры в уголь массива, иногда угольный массив упрочняется скрепляющими химическими составами.

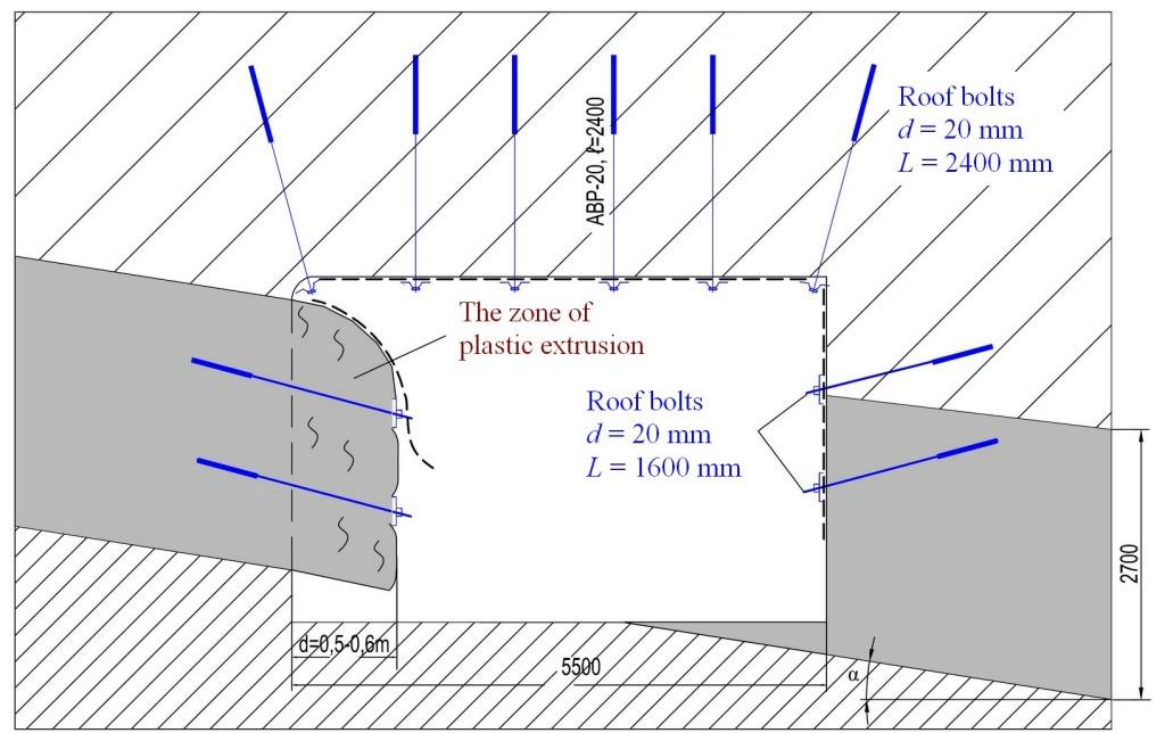

Рис. 6. Пластическое выдавливание угля и пород из бока выработки

Fig. 6. Plastic extrusion of coal and rocks from the wall of working

\section{Обоснование параметров и схем восстановления крепления подземных выработок}

Следует отметить, что действующая нормативная документация по расчету параметров и применению анкерной крепи на угольных шахтах России не охватывает вопросы ремонта поврежденных участков горных выработок. Поэтому на основе выявленных типовых форм деформаций приконтурного углепородного массива были разработаны проектные решения по усилению крепления или полной перекрепке поврежденных участков горных выработок. Они включают в себя: схему установки, обоснование параметров и номенклатуру применяемых элементов крепи-усиления, технологию работ, список применяемого оборудования. Расчет требуемых параметров крепи производился с использованием основных положений теории свода равновесия [14].

При ремонте участков выработок со скоплением отслоившихся пород кровли с деформацией решетчатой затяжкки и образованием вывалов и куполов (рис. 2) расположение куполов и вывалов относительно центра выработки определяется образовавшимся расслоением по- род или естественной слоистостью и послойными трещинами. Развитие куполов по высоте происходит по нормали к послойным трещинам и трещинам расслоения (рис. 7). При расчете параметров анкерной крепи для крепления образовавшихся при механических процессах локального образования вывалов и куполов $[15,16]$ необходимо определить следующие параметры: $B_{\text {в }}$ высота вывала; $L_{\mathrm{ST}}$ - устойчивый пролет вывала; $\delta$ - угол наклона линии облома слоев породы у опор в боках.

Ожидаемая высота вывала принимается равной высоте свода обрушения:

$$
B_{\mathrm{B}}=\frac{L}{2 \cdot K \cdot f},
$$

где $L$ - пролет выработки, м; $K$ - количество слоев от контура выработки до основания вывала $\left(K=\sum m_{i}\right) ; m_{1}$, $m_{2}, m_{3}, \mathrm{~m}_{4}$ - мощность слоев пород в кровле, м.

Устойчивый пролет вывала при углах падения пласта $\alpha$ до $10^{\circ}$ определяется по формуле:

$$
L_{\mathrm{ST}}=L-2 B_{\mathrm{B}} \cdot \operatorname{ctg} \delta,
$$

где $\delta$ - угол наклона линии облома слоев опор, град $\left(\delta=60^{\circ}-70^{\circ}\right)$. 


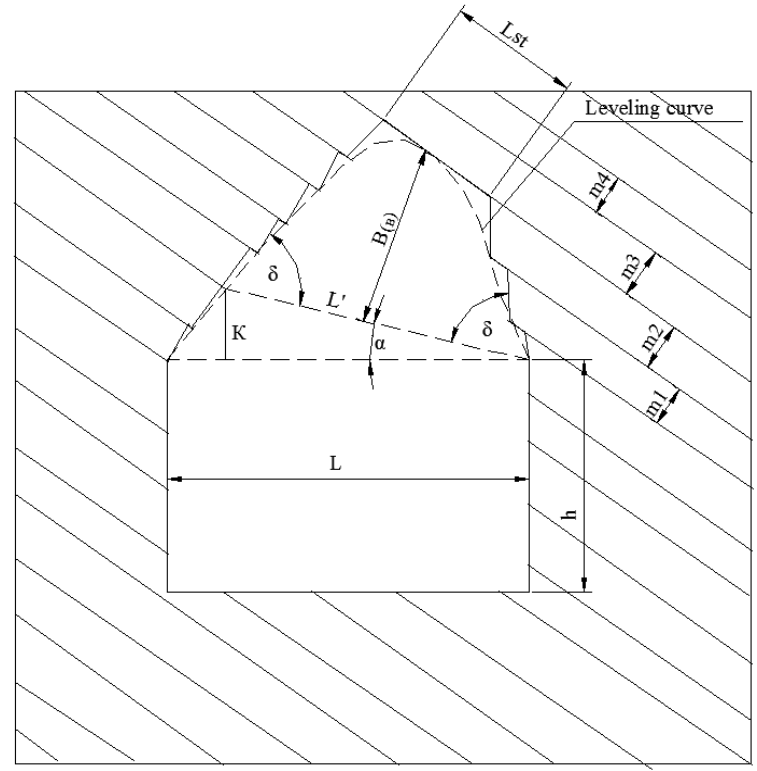

Pис. 7. Схема для определения основных ожидаемых параметров вывалов при механических прочессах локального образования вывалов или куполов

Fig. 7. Scheme for determining the main expected parameters of collapses during mechanical processes of local formation of collapses or domes

Устойчивый пролет вывала при углах падения пласта $\alpha$ более $10^{\circ}$ определяется по формуле:

$$
L_{\mathrm{ST}}=L^{\prime}-2 B_{\mathrm{B}} \cdot \operatorname{ctg} \delta,
$$

где $L^{\prime}$ - основание вывала, м.

$$
L^{\prime}=\frac{K}{\sin \alpha} .
$$

Длина анкера для крепления вывалов выработок определяется по формуле:

$$
l_{\mathrm{a}}=B_{\mathrm{B}}+l_{3},
$$

где $l_{3}$ - глубина заложения анкера за зону вывала, м (не менее 0,3 м).

При креплении вывалов схема установки анкеров может быть любая: линейная, шахматного вида, произвольная в зависимости от параметров и формы вывала. При этом целесообразно использовать плотность установки анкеров П и расстояние между анкерами $C_{\mathrm{a}}$.

Плотность установки анкеров определяется по формуле:

$$
\Pi=\frac{B_{\mathrm{B}} \cdot \gamma_{\mathrm{k}} \cdot \Pi_{\Pi}}{N_{\mathrm{a}}},
$$

где $\gamma_{\kappa}$ - объемный вес пород кровли, кН/ ${ }^{3} ; \Pi_{\Pi}-$ коэффициент перегрузки анкеров, принимается равным 1,$2 ; \mathrm{N}_{\mathrm{a}}-$ несущая способность анкера, кН.

Расстояние между анкерами определяется по формуле:

$$
\mathrm{C}_{a}=\sqrt{\frac{N_{\mathrm{a}}}{B_{\mathrm{B}} \cdot \gamma_{\mathrm{K}} \cdot \Pi_{\Pi}}} .
$$

Требуемое сопротивление для крепления вывала определяется по формуле:

$$
P_{\mathrm{a}}=B_{\mathrm{B}} \cdot \gamma_{\mathrm{K}},
$$

где $P_{\mathrm{a}}$ - сопротивление возводимой крепи в поверхности вывала, кН/ ${ }^{2}$.

Используя плотность установки анкеров и необходимое расстояние между анкерами, производится подготовка паспорта крепления выработок.

Ранее было установлено [17], что в зависимости от провисания решетчатой затяжки и ее деформации степень опасности в выработке квалифицируется следующим образом:

- $\quad$ при провисании решетчатой затяжки не более 0,3 м в выработке необходимо наладить контроль за состоянием решетчатой затяжки и выработки;

- при провисании решетчатой затяжки до 0,4 м состояние в выработке неудовлетворительное, необходимо планировать ремонтные работы;

- при провисаниях решетчатой затяжки более 0,4 м состояние в выработке следует относить к опасным, требуется производить ремонтные работы;

- при провисании решетчатой затяжки 0,5 м и более выработка находится в опасном состоянии, требуется немедленно приводить ремонтные работы.

В качестве примера реализации проектных решений по ремонту и усилению анкерного крепления выработок представлен участок вентиляционного штрека по пласту 9 в условиях ш. «Распадская» с отслоившимися породами, с разрывом решетчатой затяжки (рис. 8). Работы по ремонту необходимо производить с использованием рамной крепи - опорных рам и временной крепи. Опорная рама изготавливается из двух рудстоек диаметром не менее 200 мм с установкой стоек под деревянный верхняк. В качестве верхняка используется лафет (брус или плаха). Рама устанавливается в промежутках между анкерными рядами перед аварийным участком. Временная крепь служит для крепления части участка, где будет производиться выпуск породы из кровли и замена решетчатой затяжки.

Работы по ремонту штрека выполняются в следующей последовательности. К участку выработки доставляются элементы опорной рамы и рамы временной крепи и другие материалы - анкеры усиливающей крепи с шайбами и гайками, решетчатая затяжка, плаха (брус или лафет), переносной полок, оборудование и инструмент для выполнения работ, одна или две деревянные лестницы, металлические пики длиной не менее 3 м.

Первоначально устанавливается опорная рама с использованием распора и деревянных клиньев. После этого с помощью металлических пик проводится оборка кровли и боков от зависших кусков породы и угля. Рама временной крепи устанавливается непосредственно перед передней границей участка, где должны производиться ремонтные работы. Параметры анкеров усиливающей крепи рассчитываются по действующей в России нормативной методике, по средневзвешенному сопротивлению пород в пределах мощности, равной ширине выработки. 


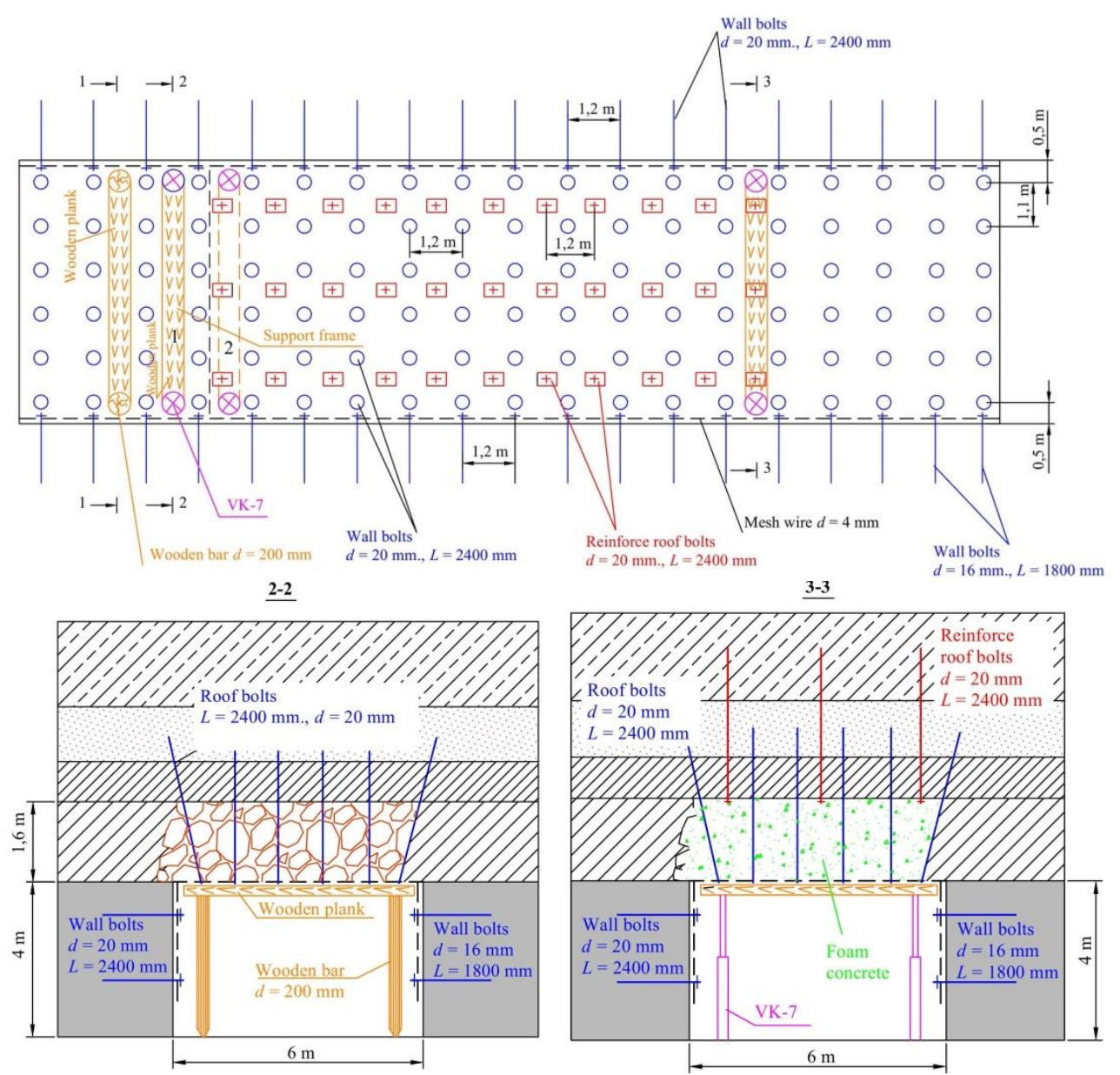

Pис. 8. Состояние штрека после выполнения ремонтных работ

Fig. 8. Roadway condition after completion of repair work

Установка анкеров осуществляется под индивидуальные подхваты в виде опорных сферических шайб с размерами $300 \times 300$ мм или плоский подхват с размерами $300 \times 300$ мм. Плотность установки анкеров не менее $0,5 \mathrm{aнк} / \mathrm{m}^{2}$. Таким образом производятся ремонтные работы по длине участка. При этом шаг выпуска пород и усиления крепи при ремонте принимается не более 1-1,2 м (рис. 8). Увеличение высоты выработки после выпуска разрушенной породы на ремонтируемом участке вызывает снижение сопротивления угля в боках штрека, увеличивает относительную напряженность в боках [18-20]. Рекомендуется увеличенную часть высоты боков в выработках после выпуска разрушенных пород кровли закреплять анкерной крепью. При этом в боках устанавливается дополнительно по одному анкеру. После установки усиливающих анкеров по всей длине участка уборки опорной рамы и рамы временной крепи производится заполнение образовавшейся пустоты легким пенобетоном.

\section{Выводы}

1. Установлено, что в процессе эксплуатации горных выработок, закрепленной анкерной крепью, в их кровле и боках происходят следующие геомеханические процессы: процесс сплошного сводообразования, процесс локальных вывалообразований и сползание боков с различной шириной призмы сползания.

2. При локальных геомеханических процессах локального вывалообразования расслоение пород, их отслоение и обрушение происходит не по всей длине выработок, а на отдельных участках выработок, где имеет место снижение прочностных свойств пород, повышенная трещиноватость, повышенная концентрация напряжений, влагонасыщение пород. На данных участках образуются вывалы пород из кровли, образование куполов, отжимы и вывалы угля из боков.

3. На основании результатов натурных исследований выявлены шесть типовых форм деформирования приконтурного углепородного массива.

4. Установлено, что в нормальных горно-геологических условиях (вне зон нарушений, вне зон ПГД) трещины давления формируются в пределах свода давления, а развитие свода происходит в направлении по нормали к создавшейся системе трещин.

5. Предложены проектные решения по усилению крепления или полной перекрепке поврежденных участков горных выработок, в которых происходят процессы локального вывалообразования. Расчет требуемых параметров крепи производился с использованием основных положений теории свода равновесия, а также на базе действующей в России нормативной методики. 


\section{СПИСОК ЛИТЕРАТУРЫ}

1. Оценка влияния динамических сейсмических воздействий на устойчивость подземных горных выработок / С.С. Цибаев, А.А. Ренев, А.С. Позолотин, С.Н. Мефодьев // Горный информационно-аналитический бюллетень. - 2020. - № 2. - С. 101-111. DOI: $10.25018 / 0236-1493-2020-2-0-101-111$

2. Mark C. Design of roof bolt systems // Proceedings: New Technology for Coal Mine Roof Support / Eds. C. Mark, D.R. Dolinar, R.J. Tuchman, T.M. Barczak, S.P. Signer, P.F. Wopat. - Cincinnati, OH: U.S. Department of Health and Human Services, Public Health Service, Centers for Disease Control and Prevention, National Institute for Occupational Safety and Health, 2000. - P. 99-109. URL: https://stacks.cdc.gov/ view/cdc/8306 (дата обрашения: 14.03.2021).

3. Shreedharan S., Kulatilake P.H.S.W. Discontinuum - equivalen continuum analysis of the stability of tunnels in a deep coal mine using the distinct element method // Rock Mechanics And Rock Engineering. - 2016. - № 49 (5). - P. 1903-1922. URL https://www.researchgate.net/publication/284103706 DiscontinuumEquivalent_Continuum_Analysis_of_the_Stability_of_Tunnels_in a_Deep_Coal_Mine_Using_the_Distinct_Element_Method (дата обращения: 14.03.2021)

4. Factors influencing intersection stability in U.S. coal mines G.M. Molinda, C. Mark, E.R. Bauer, D.R. Babich, D.M. Pappas // Proceedings of the 17th International Conference on Ground Control in Mining / ed. by S.S. Peng. - Morgantown, WV University of West Virginia, 1998. - P. 267-275. URL: https://www.cdc.gov/niosh/mining/works/coversheet599.html (дата обращения: 14.03.2021)

5. Analysis of rock mass and anchor support elements deformations during the long-term maintenance of mine workings / S. Tsibaev, A. Renev, R. Zainulin, A. Kucherenko // Vth Internationa Innovative Mining Symposium. - 2020. - V. 174. URL: https://doi.org/10.1051/e3sconf/202017401001 (дата обращения: 14.03.2021).

6. Renev A., Tsibaev S., Kalinin S. The evaluation of negative anthropogenic factors subjection on bolts stability and surrounding massif deformations // Coal in the 21st Century: Mining, Intelligent Equipment and Environment Protection: 9th ChinaRussia Symposium. - 2018. - P. 359-364. URL: https://doi.org/ 10.2991/coal-18.2018.67 (дата обращения: 14.03.2021).

7. Rock pressure manifestation in development workings advanced in a thick coal seam / K. Filimonov, D. Zorkov, S. Tsibaev, A. Kucherenko // Vth International Innovative Mining Symposium. - 2020. - V. 174. URL: https://doi.org/10.1051/e3sconf/202017401037 (дата обращения: 14.03.2021).

8. Abramovich A., Pudov E., Kuzin E. Prerequisites for the establishment of the auto-mated monitoring system and accounting of the displacement of the roof of underground mines for the improvement of safety of mining work // The Second International Innovative Mining Symposium. E3C Web of Conferences. 2017. - V. 21. URL: https://www.researchgate.net/publication/ 320985259_Prerequisites_for_the_Establishment_of_the_Automat ed_Monitoring_System_and_Accounting_of_the_Displacement_o f_the_Roof_of_Underground_Mines_for_the_Improvement_of_Sa fety_of_Mining_Work (дата обращения: 14.03.2021).

9. Рекомендации по систематическому визуальному контролю за работой анкерной крепи / В.П. Баскаков, Н.Т. Бедарев, С.Г. Костюк и др. - Прокопьевск: КузГТУ, 2013. - 39 с.

10. Practical investigations into resin anchored roof bolting parameters / J. Purcell, D. Vandermaat, M. Callan, P. Craig // Proceedings of the 16th Coal Operators' Conference, Mining Engineering. Wollongong (NSW): University of Wollongong, 2016. - P. 53-63.

11. Colback P., Wiid B.L. The influence of moisture content on the compressive strength of rocks // Proceedings of the 3rd Canadian Rock Mechanism Symposium. - Toronto: University of Toronto, 1965. - P. 65-83.

12. Influence of water content and anisotropy on the strength and deformability of low porosity meta-sedimentary rocks under triaxial compression / D. Li, L.N.Y. Wong, G. Liu, X. Zhang // Engineering Geology. - 2012. - V. 126. - P. 46-66. URL: https://www.researchgate.net/publication/256699765_Influence_of _water_content_and_anisotropy_on_the_strength_and_deformabili ty_of_low_porosity_meta-

sedimentary_rocks_under_triaxial_compression (дата обращения: 14.03.2021)

13. Practical approaches to sand management / A. Acock, T. Orourke, D. Shirmboh, J. Alexander, G. Andersen, T. Kaneko, A. Venkitaraman, J. Lopez de Cardenas, M. Nishi, M. Numasawa, K. Yoshioka, A. Roy, A. Wilson, A. Twynam // Oilfield Review. 2004. - V. 16. - P. 10-27. URL : https://www.researchgate.net/ publication/288809656_Practical_approaches_to_sand_manageme nt (дата обращения: 14.03.2021)

14. Цимбаревич П.М. Механика горных пород. - М.: Углетехиздат, 1948. - $184 \mathrm{c}$.

15. Gale W.J., Fabjanczyk M.W., Guy R.J. Optimization of reinforcement design of coal mine roadways // Proceedings of the 11th International Conference on Ground Control in Mining. Wollongong, New South Wales, Australia: University of Wollongong, 1992. - P. 272-279.

16. Esterhuizen G.S., Tulu I.B. Analysis of alternatives for using cable bolts as primary support at two low-seam coal mines // International Journal of Mining Science and Technology. 2015. - V. 16. URL: https://www.researchgate.net/publication/ 287374864_Analysis_of_alternatives_for_using_cable_bolts_as_p rimary_support_at_two_low-seam_coal_mines (дата обращения: 14.03.2021).

17. Исследование влияния очистных работ на состояние штреков, поддерживаемых в целиках в условиях шахты «ИМ. В.Д. Ялевского» / К.А. Филимонов, А.А. Ренёв, А.В. Кучеренко, П.В. Гречишкин // Горный информационно-аналитический бюллетень. - 2019. - № 5. - С. 133-148. DOI: 10.25018/02361493-2019-05-0-133-148.

18. Zhu W., Xu J., Xu G. Mechanism and control of roof fall and support failure incidents occurring near longwall recovery roadways // The Journal of the Southern African Institute of Mining and Metallurgy. - 2017. - V. 117. - № 11. - P. 1063-1072. DOI: $10.17159 / 2411-9717 / 2017 /$ v117n11a11.

19. Karampinos E., Hadjigeorgiou J., Pierce M. Explicit representation of rock reinforcement in 3D DEM models for foliated ground // The Journal of the Southern African Institute of Mining and Metallurgy. - 2018. - V. 118. - № 12. - P. 1243-1250. DOI: $10.17159 / 2411-9717 / 2018 /$ v118n12a2.

20. Numerical study on roadway stability under weak geological condition of PT Gerbang Daya Mandiri Underground Coal Mine in Indonesia / P. Phanthoudeth, T. Sasaoka, H. Shimada, B. Ulaankhuu, J. Oya, S. Dwiki, T. Karian // GSTF Journal of Geological Sciences (JGS). - 2016. - V. 3. - № 1. - P. 15-23. DOI: $10.5176 / 2335-67743.1 .26$.

Поступила: 28.04 .20212$.

\section{Информация об авторах}

Цибаев С.C., кандидат технических наук, доцент кафедры разработки месторождений полезных ископаемых Кузбасского государственного технического университета имени Т.Ф. Горбачева.

Ренев A.A., доктор технических наук, профессор, заведующий кафедрой разработки месторождений полезных ископаемых Кузбасского государственного технического университета имени Т.Ф. Горбачева.

Ванг Цин, профессор, государственная лаборатория предотвращения и контроля катастроф в горном деле Шаньдунского научно-технического университета.

Юй Фэн-Хай, научный сотрудник, государственная лаборатория предотвращения и контроля катастроф в горном деле Шаньдунского научно-технического университета. 
UDC 622.281 .74

\title{
ANALYSIS OF DEFORMATION OF TYPICAL FORMS SURROUNDING ROCK MASSIF FOR MINE WORKINGS SUPPORT RECOVERY
}

\author{
Sergey S. Tsibaev ${ }^{1}$, \\ cibaevss@kuzstu.ru
}

\author{
Alexey A. Renev 1 , \\ raa@kuzstu.ru \\ Qing-duo Wan², \\ qwang@sdust.edu.cn \\ Feng-hai $Y u^{2}$, \\ yufenghai2006@163.com \\ 1 T.F. Gorbachev Kuzbass State Technical University, \\ 28, Vesennyaya street, Kemerovo, 650000, Russia. \\ 2 Shandong University of Science and Technology, \\ 579 Qianwangang Road, Huangdao District, Qingdao, Shandong Province, 266590, China.
}

Relevance. More than $75 \%$ of underground mine workings are supported by anchorage nowadays. In the case of long-term mine workings maintenance the deformations of surrounding rock massif and anchoring elements occur. The surrounding rock massif typical forms deformations determination in order to develop the design solutions and substantiate anchorage parameters is currently urgent scientific and practical task. Especially it is important in case of mine workings support repair or recovery stages.

The main aim is mine working anchorage design solutions and parameters substantiate during the repair and recovery stages.

Methods: field measurements, statistical processing of results.

Results. The paper introduces the description, causes of occurrence and manifestation forms of geomechanical processes in surrounding rock massif supported by anchorage. Based on the long-term field measurements six forms of rock massif typical deformations have been established. The roof bridging begins with decompaction of rocks, which causes rocks stratification under the influence of gravity. This results in cavities formation and lower layers separation from upper ones. Because of formation of the plastic deformation zone around mine workings the extraction and dumping coal from walls occur. Coal and rocks are in an unbound state and are kept in balance due to the forces of friction and resistance of the support in the zones of plastic deformations. Its width depends on many factors such as the working width and the internal friction angle. The coefficient of stress concentration in the walls rises up due to growth of mine working widht.

\section{Key words:}

Mine workings stability, mine workings repair, anchorage, deformations, displacement, convergence.

\section{REFERENCES}

1. Tsibaev S.S, Renev A.A, Pozolotin A.A, Mefodiev S.N. Assessment of seismic impacts on stability of openings in underground mines. Mining informational and analytical bulletin (scientific and technical journal), 2020, no. 2, pp. 101-111. DOI: 10.25018/02361493-2020-2-0-101-111 In Rus.

2. Mark C. Design of roof bolt systems. Proceedings: New Technology for Coal Mine Roof Support. Eds. C. Mark, D.R. Dolinar, R.J. Tuchman, T.M. Barczak, S.P. Signer, P.F. Wopat. Cincinnati, OH, U.S. Department of Health and Human Services, Public Health Service, Centers for Disease Control and Prevention, National Institute for Occupational Safety and Health, 2000. pp. 99-109. Available at: https://stacks.cdc.gov/view/cdc/8306 (accessed 14 March 2021)

3. Shreedharan S., Kulatilake P.H.S.W. Discontinuum - equivalen continuum analysis of the stability of tunnels in a deep coal mine using the distinct element method. Rock Mechanics and Rock Engineering, 2016, no. 49 (5), pp. 1903-1922. Available at: https://www.researchgate.net/publication/284103706_DiscontinuumEquivalent_Continuum_Analysis_of the_Stability_of_Tunnels_in a_Deep_Coal_Mine_Using_the_Distinct_Element_Method (accessed 14 March 2021).

4. Molinda G.M., Mark C., Bauer E.R., Babich D.R., Pappas D.M Factors Influencing Intersection Stability in U.S. Coal Mines. Proceedings of the $17^{\text {th }}$ International Conference on Ground Control in Mining. Ed. by S.S. Peng. Morgantown, WV, University of West Virginia, 1998. pp. 267-275. Available at https://www.cdc.gov/niosh/mining/works/coversheet599.html (accessed 14 March 2021).
5. Tsibaev S., Renev A., Zainulin R., Kucherenko A. Analysis of rock mass and anchor support elements deformations during the long-term maintenance of mine workings. $V^{\text {th }}$ International Innovative Mining Symposium, 2020, vol. 174. Available at: https://doi.org/10.1051/e3sconf/202017401001 (accessed 14 March 2021)

6. Renev A., Tsibaev S., Kalinin S. The evaluation of negative anthropogenic factors subjection on bolts stability and surrounding massif deformations. $9^{\text {th }}$ China-Russia Symposium. Coal in the $21^{\text {st }}$ Century: Mining, Intelligent Equipment and Environment Protection, 2018, pp. 359-364. Available at: https://doi.org/ 10.2991/coal-18.2018.67 (accessed 14 March 2021).

7. Filimonov K., Zorkov D., Tsibaev S., Kucherenko A. Rock pressure manifestation in development workings advanced in a thick coal seam. $V^{\text {th }}$ International Innovative Mining Symposium, 2020, vol. 174. Available at: https://doi.org/10.1051/e3sconf/ 202017401037 (accessed 14 March 2021).

8. Abramovich A., Pudov E., Kuzin E. Prerequisites for the establishment of the auto-mated monitoring system and accounting of the displacement of the roof of underground mines for the improvement of safety of mining work. The Second International Innovative Mining Symposium. E3C Web of Conferences, 2017, vol. 21. Available at: https://www.researchgate.net/publication/ 320985259_Prerequisites_for_the_Establishment_of_the_Automat ed_Monitoring_System_and_Accounting_of_the_Displacement_o f_the_Roof_of_Underground_Mines_for_the_Improvement_of_Sa fety_of_Mining_Work (accessed 14 March 2021).

9. Baskakov V.P. Rekomendatsii po sistematicheskomu vizualnomu kontrolyu za rabotoy ankernoy krepi [The bolts control visual me- 
thodical recommendation]. Prokopevsk, KuzGTU Publ., 2013. $39 \mathrm{p}$.

10. Purcell J., Vandermaat D., Callan M., Craig P. Practical investigations into resin anchored roof bolting parameters. Proceedings of the $16^{\text {th }}$ Coal Operators' Conference, Mining Engineering. Wollongong (NSW), University of Wollongong, 2016. pp. 53-63.

11. Colback P., Wiid B.L. The influence of moisture content on the compressive strength of rocks. Proceedings of the $3^{\text {rd }}$ Canadian Rock Mechanism Symposium. Toronto, University of Toronto, 1965. pp. 65-83.

12. Li D., Wong L.N.Y., Liu G., Zhang X. Influence of water content and anisotropy on the strength and deformability of low porosity meta-sedimentary rocks under triaxial compression. Engineering Geology, 2012, vol. 126, pp. 46-66. Available at: https://www.researchgate.net/publication/256699765_Influence_of _water_content_and_anisotropy_on_the_strength_and_deformabili ty_of_low_porosity_meta-sedimentary_rocks_under_triaxial_ compression (accessed 14 March 2021).

13. Acock A., Orourke T., Shirmboh D., Alexander J., Andersen G., Kaneko T., Venkitaraman A., Lopez de Cardenas J., Nishi M., Numasawa M., Yoshioka K., Roy A., Wilson A., Twynam A. Practical approaches to sand management. Oilfield Review, 2004 vol. 16, pp. 10-27. Available at: https://www.researchgate.net/ publication/288809656_Practical_approaches_to_sand_manageme nt (accessed 14 March 2021).

14. Tsimbarevich P.T. Mekhanika gornykh porod [Mine rock mechanics]. Moscow, Ugletekhizdat Publ., 1948. 184 p.

15. Gale W.J., Fabjanczyk M.W., Guy R.J. Optimization of reinforcement design of coal mine roadways. Proceedings of the $11^{\text {th }}$ International Conference on Ground Control in Mining. Wollon- gong, New South Wales, Australia, University of Wollongong, 1992. pp. 272-279.

16. Esterhuizen G.S., Tulu I.B. Analysis of alternatives for using cable bolts as primary support at two low-seam coal mines. International Journal of Mining Science and Technology, 2015, vol. 16. Available at: https://www.researchgate.net/publication/287374864 Analysis_of_alternatives_for_using_cable_bolts_as_primary support at two low-seam coal mines (accessed: 14 March 2021).

17. Filimonov K.A., Renev A.A, Kucherenko A.V., Grechishkin P.V. Influence of longwalling on reinforced roadways in pillars in Yalevsky Mine. Mining informational and analytical bulletin (scientific and technical journal), 2019, no. 5, pp. 133-148. DOI: 10.25018/0236-1493-2019-05-0-133-148. In Rus.

18. Zhu W., Xu J., Xu G. Mechanism and control of roof fall and support failure incidents occurring near longwall recovery roadways. The Journal of the Southern African Institute of Mining and Metallurgy, 2017, vol. 117, no. 11, pp. 1063-1072. DOI: 10.17159/2411-9717/2017/v117n11a11

19. Karampinos E., Hadjigeorgiou J., Pierce M. Explicit representation of rock reinforcement in 3D DEM models for foliated ground. The Journal of the Southern African Institute of Mining and Metallurgy, 2018, vol. 118, no. 12, pp. 1243-1250. DOI: 10.17159/24119717/2018/ v118n12a2

20. Phanthoudeth P., Sasaoka T., Shimada H., Ulaankhuu B., Oya J., Dwiki S., Karian T. Numerical study on roadway stability under weak geological condition of PT Gerbang Daya Mandiri underground coal mine in Indonesia. GSTF Journal of Geological Sciences (JGS), 2016, vol. 3, no 1, pp. 15-23. DOI: 10.5176/233567743.1 .26

Received: 28 April 2021.

\section{Information about the authors}

Sergey S. Tsibaev, Cand. Sc., associate professor, T.F. Gorbachev Kuzbass State Technical University. Alexey A. Renev, Dr. Sc., professor, head of the department, T.F. Gorbachev Kuzbass State Technical University.

Qing-duo Wan, professor, State Key Laboratory of Mining Disaster Prevention and Control Co-founded by Shandong Province and the Ministry of Science and Technology, Shandong University of Science and Technology.

Feng-hai Yu, researcher, State Key Laboratory of Mining Disaster Prevention and Control Co-founded by Shandong Province and the Ministry of Science and Technology, Shandong University of Science and Technology. 